\title{
Integrating BCC with existing ICT System for TB: Nudge Early Detection and Treatment of Latent Tuberculosis in India- A Short Communication
}

\author{
Nilofur Banu
}

\section{Nilofur Banu}

Consultant Epidemiologist, Preventive Medicine Department, Vijaya Medical and Educational Trust, Vijaya group of Hospitals, Chennai, Tamil Nadu, INDIA.

\section{Correspondence}

\section{Dr. Nilofur Banu}

Consultant Epidemiologist, Preventive medicine Department, Vijaya Medical and Educational Trust, Vijaya group of Hospitals, Chennai, Tamil Nadu, INDIA. Email: dr.nilofurbanuu@gmail.com

\section{History}

- Submission Date: 25-10-2021

- Revised Date: 17-11-2021

- Accepted Date: 20-12-2021

DOI : 10.5530/ijmedph.2022.1.8

Article Available online

http://www.ijmedph.org/v12/i1

\section{Copyright}

(C) 2022 Phcog.Net. This is an openaccess article distributed under the terms of the Creative Commons Attribution 4.0 International license.

\begin{abstract}
To eliminate tuberculosis, One of the main subjects to be focused at present is Latent Tuberculosis Infection (LTBI) because it causes persistence of immunological response in humans to Mycobacterium tuberculosis antigen without manifesting any clinical sign and symptoms of active tuberculosis. If LTBI is not diagnosed and treated, it has been estimated that one in ten people with LTBI will get sick with tuberculosis infection. It has been a challenge to convince the contact and the target group to undergo screening as both TST and IGRA are an invasive procedure and to convince in apparently healthy (without any symptoms) individual to undergo screening is a foremost Potential barrier in the diagnosis of LTBI and initiating preventive treatment. The screening strategies must be coincide with appropriate individual (who are eligible candidate) and community level education to avoid any undesired harm for tested individuals, this can be achieved by Behavioral Change Communication (BCC). For more effective medium or channels to improve rate by utilizing and Nikshay portal may serve as an effective channel for BCC for early detection of latent tuberculosis and preventive treatment as it has suitable environment and support system to improve the success rate and to eliminate tuberculosis.

Key words: Latent tuberculosis, Behavioral Change Communication, Integration with ICT, Nikshay portal, Tuberculosis elimination, Preventive tuberculosis treatment.
\end{abstract}

\section{Introduction - Significant of latent tuberculosis infection}

Globally, lots of effects have been taken to eliminate tuberculosis through many strategies, program which includes early detection and treatment of active tuberculosis infection which is the major intervention pertaining to the elimination of tuberculosis, but still one of the main subjects to be focused in this regard because Latent Tuberculosis Infection (LTBI) is due to persistence of immunological response in humans to Mycobacterium tuberculosis antigen without manifesting any clinical sign and symptoms of active tuberculosis. ${ }^{1}$ If LTBI is not diagnosed and treated, it has been estimated that one in ten people with LTBI will get sick with tuberculosis infection and the risk is much higher among HIV infected person and in young children, as it has been reported that if LTBI are untreated in children's it will cause $40 \%$ active tuberculosis infection in children under 1 year of age, $24 \%$ in children between the age group of $1-10$ years and $16 \%$ in those belong to $11-15$ years. ${ }^{2-4}$ Hence LTBI represent a reservoir of tuberculosis bacilli and therefore it will be difficult to eliminate tuberculosis, therefore there must be a paradigm shift towards aggressive diagnosis and treatment of LTBI is necessary to eliminate tuberculosis.
Identifying target population for initiating TB Preventive treatment (TPT)

Identification and initiation of treatment are done by targeting approach as it is considered as a relevant public health response and this is done by prioritizing the target population for TPT based on speculative risk of development of latent infection to active TB or else based on the increased likelihood of exposure to active TB disease, the eligible group includes children $>5$ years, People living with HIV (which includes Adults and children $>12$ months and Infants $<12$ months with HIV in contact with active $\mathrm{TB})$, All household contacts of active pulmonary TB patients and Individuals with clinical risk factors like on immunosuppressive therapy, having silicosis, on dialysis, an anti-TNF treatment, preparing for hematologic or organ transplantation and the next step after identifying the target population is confirming that target population has not developed active tuberculosis infection because this give the confidence to the provider as well as satisfaction for the eligible population. ${ }^{5}$ Till recent date, there is no gold standard investigation to anticipate progression to TB disease among those infected, currently recommend investigation are Tuberculin Skin Test (TST) and Interferon-Gamma Release Assay (IGRA), both these tests measure immune
Cite this article : Banu N. Integrating BCC with existing ICT System for TB: Nudge Early Detection and Treatment of Latent Tuberculosis in India- A Short Communication. Int J Med Public Health. 2022;12(1):36-8. 
sensitization to mycobacterial protein antigens that occurs following infection by $M$. tuberculosis and these test are invasive methods to rule out active tuberculosis disease among the target population, Following the confirmation of LTBI preventive treatment will be started to the eligible population. ${ }^{6}$

\section{Challenges in identifying and initiating preventive treatment for LTBI}

Though the protocol and guidelines are very clear it has been a challenge to convince the contact and the target group to undergo screening as both TST and IGRA are an invasive procedure and to convince in apparently healthy (without any symptoms) individual to undergo screening is a foremost Potential barrier in the diagnosis of LTBI and initiating preventive treatment, ${ }^{7}$ many aggressive steps have been taken to increase the overall screening and preventive treatment for TB, like counseling of the index case and thereby contacts and high risk individual, though health care professionals but still many target population does not undergo screening because of the Stigma, which is another potential impact of routine screening for LTBI, in an asymptomatic individual, more over the majority of target individuals with a positive TST or IGRA will never develop active $\mathrm{TB}$ and in those cases the screening causes anxiety and prejudice, ${ }^{8}$ It has been reported that only $70 \%$ of eligible candidate are willing for screening and the eligible candidates only $62 \%$ of them undergo testing, $56 \%$ of the eligible population are referred for test, $30 \%$ of the eligible candidate receive treatment but only $18 \%$ of the eligible candidate receive treatment, but only $18 \%$ of the eligible candidate finished the treatment completely despite of counseling by health care workers. ${ }^{9-10}$

\section{Addressing Challenges by Integrating Behavioral change communication with existing ICT system for TB to overcome the barriers}

It is noteworthy from the challenges faced for screening and preventive treatment that the screening strategies must be coincide with appropriate individual (who are eligible candidate) and community level education to avoid any undesired harm for tested individuals, this can be achieved by Behavioral Change Communication (BCC) which is a part of broader sub-discipline of health communication which in turn is the study and application of various communication strategies for the purpose of promoting positive health outcomes. ${ }^{11}$

Eventually BCC leads to a participatory process by motivating, positive health behavior change in eligible individuals and communities and this can be achieved by the strategic approach of targeted tailor made messages, and thereby providing a supportive environment. ${ }^{11}$ Primarily a positive attitude change is what's needed among the target population for the acceptance to undergo screening and to initiate and complete the preventive TB treatment and in order to reach the target population communication channels are frequently employed and these channels may range from one -on-one (interpersonal under the program by health care workers) to various electronic channels. ${ }^{12}$ Choosing the appropriate communication channel is also very important because the channels have the ability and capacities to reach target audiences, far better than conventional methods. ${ }^{13,14}$ Thus, if well-developed information and communication technology paired with evidence based behavior change communications may influence behavioral change across various drivers irrespective of population groups, age, race, location and education. ${ }^{15,16}$ Currently, the existing Nikshay portal (an information and communication technology, which is the web enabled patient management system for TB control under the National Tuberculosis Elimination Program) has upgraded to a person's lifecycle approach with strategically positioning for The entry point for information of individuals who are stated as the target population into the TPT care cascade of screening, testing, TPT initiation, eligibility assessment, compliance towards treatment and to follow-up till treatment are completed this is provided with much needed technical support like Nikshay Sampark tele caller to address the queries..$^{10}$ By collaborating behavioral change communication through the existing Nikshay portal as a channel for communication to the target audience, it will capture the felt need and will be able to address the same. The interactive potential of Nikshay portal will be suitable for effective BCC and based on the understanding BCC is not just the mere transmission of health information to target audience, but a make Unaware to become Aware, Concerned, Knowledgeable eventually motivated to change and sustained those changes, the multi-way interactivity with modern information and communication technologies offers an unmatched advantage ${ }^{17}$ Nikshay portal has the platform for peer support, social along with emotional support this qualities are perhaps the most essential requisites for BCC, ${ }^{18}$ Lastly BCC along with suitable environment and advance technology in a Nikshay portal like Geo tagging and availability of testing and treatment centers will not only be helpful to overcome barriers, but will also help with compliance towards treatment and together will be able to eliminate tuberculosis.

\section{CONCLUSION}

As BCC is a core part of the strategies deployed by public health because mere health awareness and availability of relevant information may not necessarily bring changes in health behavior or acceptance of new screening practices or may not lead to initiation of treatment and compliance towards treatment. Despite of effort taken by health care workers, policy makers, inter sectoral coordination and public private association desirable results in terms of early diagnosis and initiation of treatment for latent tuberculosis were not achieved, Which had led to lookout for more effective medium or channels to improve rate by utilizing and Nikshay portal may serve as an effective channel for BCC for early detection of latent tuberculosis and preventive treatment as it has suitable environment and support system to improve the success rate and to eliminate tuberculosis.

This may also require adequate planning and strategies in Nikshay portal and BCC to be incorporated should be evidence based, capturing the client's perspective by addressing felt need by research with epidemiological evidence for more effective collaboration and achieving desirable results.

\section{CONFLICT OF INTEREST}

The author declares that there is no conflict of interest.

\section{REFERENCES}

1. Mack U, Migliori GB, Sester M, Rieder HL, Ehlers S, Goletti D, et al. Lange, TBNET. LTBI: Latent tuberculosis infection or lasting immune responses to M. tuberculosis? A TBNET consensus statement. Eur Respir J. 2009 May 1;33(5):956-73. doi: 10.1183/09031936.00120908, PMID 19407047

2. Comstock GW, LivesayVT, WOOLPERT SF. The prognosis of a positive tuberculin reaction in childhood and adolescence. Am J Epidemiol. 1974 Feb 1;99(2):131-8. doi: 10.1093/oxfordjournals.aje.a121593, PMID 4810628.

3. Reichman LB, Hershfield ES. Tuberculosis: A comprehensive international approach. CRC Press; 2000 Mar 8.

4. Saha S, Kumar A, Saurabh K, Shankar SH, Kashyap A, Nischal N, et al. Current status of treatment of latent tuberculosis infection in India. Indian J Med Sci. 2020 Feb 25;71(2):54-9. doi: 10.25259/IJMS_18_2019.

5. Paradkar M, Padmapriyadarsini C, Jain D, Shivakumar SVBY, Thiruvengadam K Gupte AN, et al. CTRIUMPH-RePORT India Study Team. Tuberculosis preventive treatment should be considered for all household contacts of pulmonary tuberculosis patients in India. PLOS ONE. 2020 Jul 29;15(7):e0236743. doi: 10.1371/journal.pone.0236743, PMID 32726367.

6. Lewinsohn DM, Leonard MK, LoBue PA, Cohn DL, Daley CL, Desmond E, et al. Official American Thoracic Society/Infectious Diseases Society of 
America/Centers for Disease Control and Prevention clinical practice guidelines: Diagnosis of tuberculosis in adults and children. Clin Infect Dis. 2017 Jan 15;64(2):e1-e33. doi: 10.1093/cid/ciw694, PMID 27932390.

7. Ruhwald M, Aggerbeck H, Gallardo RV, Hoff ST, Villate JI, Borregaard B, et al. TESEC Working Group. Safety and efficacy of the C-Tb skin test to diagnose Mycobacterium tuberculosis infection, compared with an interferon $\gamma$ release assay and the tuberculin skin test: A phase 3, double-blind, randomised, controlled trial. Lancet Respir Med. 2017;5(4):259-68. doi: 10.1016/S22132600(16)30436-2, PMID 28159608.

8. Banerjee U, Sankar S, Singh A, Chandra N. A Multi-Pronged Computational Pipeline for Prioritizing Drug Target Strategies for Latent Tuberculosis. Frontiers in chemistry. 2020;8.doi: 10.3389/fchem.2020.593497

9. Carranza C, Pedraza-Sanchez S, De Oyarzabal-Mendez E, Torres M. Diagnosis for latent tuberculosis infection: New alternatives. Front Immunol. 2020;11:2006. doi: 10.3389/fimmu.2020.02006, PMID 33013856.

10. Ministry of Health and Family Welfare. Guidelines for programmatic management of TB preventive. India; 2021 Jul. p. 90.

11. 1 Kreps GL, Maibach EW. Transdisciplinary science: the nexus between communication and public health. J Commun. 2008 Dec 1;58(4):732-48. doi: 10.1111/j.1460-2466.2008.00411.x.

12. Ogata Jones K, Denham BE, Springston JK. Effects of mass and interpersonal communication on breast cancer screening: advancing agenda-setting theory in health contexts This article is drawn from data gathered for the doctoral dissertation of Karyn Ogata Jones at the University of Georgia in 2003. An earlier version of this article was presented at the National Communication Association Annual Conference, Health Communication Division, November 2005. J Appl Commun Res. 2006 Feb 1;34(1):94-113. doi: 10.1080/00909880500420242.

13. Moorhead SA, Hazlett DE, Harrison L, Carroll JK, Irwin A, Hoving C. A new dimension of health care: systematic review of the uses, benefits, and limitations of social media for health communication. J Med Internet Res. 2013 Apr 23;15(4):e85. doi: 10.2196/jmir.1933, PMID 23615206

14. Keller JM. Motivational design for learning and performance: The ARCS model approach. Springer Science+Business Media; 2009 Nov 24.

15. Kukreja P, Heck Sheehan AH, Riggins J. Use of social media by pharmacy preceptors. Am J Pharm Educ. 2011 Nov 10;75(9):176. doi: 10.5688/ajpe759176, PMID 22171104.

16. Scanfeld D, Scanfeld V, Larson EL. Dissemination of health information through social networks: Twitter and antibiotics. Am J Infect Control. $2010 \mathrm{Apr}$ 1;38(3):182-8. doi: 10.1016/j.ajic.2009.11.004, PMID 20347636.

17. Schoenborn CA, Adams PE. Health behaviors of adults: United States, 20052007. Vital Health Stat 10. 2010 Mar 1;245(245):1-132. PMID 20669609.

18. O'Dea B, Campbell A. Healthy connections: online social networks and their potential for peer support. Stud Health Technol Inform. 2011;168:(133-40). PMID 21893921.

Cite this article : Banu N. Integrating BCC with existing ICT System for TB: Nudge Early Detection and Treatment of Latent Tuberculosis in India- A Short Communication. Int J Med Public Health. 2022;12(1):36-8. 